\title{
Use of very low tidal volumes during high-frequency ventilation reduces ventilator lung injury
}

\author{
Noelia González-Pacheco ${ }^{1}$ Manuel Sánchez-Luna ${ }^{1}$ Paz Chimenti-Camacho ${ }^{1} \cdot$ Martín Santos-González $^{2}$. \\ Paula Palau-Concejo ${ }^{3} \cdot$ Francisco Tendillo-Cortijo $^{2}$
}

Received: 26 July 2018 / Revised: 13 December 2018 / Accepted: 28 January 2019 / Published online: 15 February 2019

(c) Springer Nature America, Inc. 2019

\begin{abstract}
The use of volume guarantee (VG) on high-frequency oscillatory ventilation (HFOV) allows to use fixed very low highfrequency tidal volume (VThf), maintaining adequate $\mathrm{CO}_{2}$ removal while potentially reducing the risk of ventilator-induced lung injury.

Objective To demonstrate that the use of very low VThf can be protective compared with standard VThf on HFOV combined with VG in a neonatal animal model.

Study design Experimental study in 2-day-old piglets with induced respiratory distress syndrome ventilated with two different HFOV strategies combined with VG $\left(10 \mathrm{~Hz}\right.$ with high VThf versus $20 \mathrm{~Hz}$ with very low VThf at similar PaCO $\mathrm{Ca}_{2}$ ). After $12 \mathrm{~h}$ of mechanical ventilation, the pulmonary histologic pattern was analyzed.

Results We found in the $10 \mathrm{~Hz}$ group with the higher VThf compared with the $20 \mathrm{~Hz}$ and very low VThf group more evident and more severe histological lesions with inflammatory infiltrate within the alveolar wall and alveolar space, as well as large areas of parenchyma consolidation and areas of alveolar hemorrhage in the more severe cases.

Conclusion The use of very low VThf compared with higher VThf at similar $\mathrm{CO}_{2}$ removal reduces lung injury in a neonatal animal model of lung injury after prolonged mechanical ventilation with HFOV combined with VG.
\end{abstract}

\section{Introduction}

The pathogenesis of bronchopulmonary dysplasia (BPD) is multifactorial and is characterized by an arrest of the normal growth and development of the immature lung, and lung injury due to oxygen exposure, "ventilator-induced lung injury" (VILI) and prenatal and postnatal inflammation [1]. VILI is multifactorial, and is believed to be due to a combination of volutrauma and atelectotrauma [2]. Furthermore,

Manuel Sánchez-Luna

msluna@salud.madrid.org

1 Neonatology Department, Hospital Universitario Gregorio Marañón, Biomedical Research Institute Gregorio Marañón, Complutense University of Madrid, Madrid, Spain

2 Medical and Surgical Research Unit, Instituto de Investigación Sanitaria Puerta de Hierro, Hospital Universitario Puerta de Hierro, Majadahonda, Madrid, Spain

3 Servicio de Inmunología, Laboratorio de Inmuno-Biología Molecular, Hospital Universitario Gregorio Marañón, Madrid, Spain overdistension of the lung stimulates the release of multiple products that promote an inflammatory response. Subsequently, these proinflammatory cytokines attract peripheral leukocytes into the lung amplifying preexisting lung injury and interfering with alveolarization (biotrauma) [3, 4].

It is well known that VILI may be reduced by the use of gentle ventilation strategies based on reducing the magnitude and duration of mechanical ventilation; one of these ventilation strategies consists of using faster ventilator rate to decrease as much as possible tidal volume and to reduce volutrauma and the release of inflammatory mediators, while achieving adequate gas exchange [5, 6].

High-frequency oscillatory ventilation (HFOV) was developed as a promising ventilation technique to decrease VILI, using tidal volumes smaller than anatomical dead space, delivered at frequencies above normal physiological breathing, and superimposed on a continuous positive pressure [7]. Unlike initial results in animal models [8, 9], the use of elective HFOV compared with conventional mechanical ventilation (CMV) has not succeeded in demonstrating significant differences in decreasing the risk of BPD in preterm infants. This lack of difference has 
presumably been attributed to the use of different interventions and individual patients across trials [10] and, what more importantly, to the use of devices that did not measure high-frequency tidal volume (VThf) that might have been larger than the anatomical dead space [11].

During $\mathrm{HFOV}, \mathrm{CO}_{2}$ removal is directly proportional to the square of the VThf and the frequency (fR) by the following equation: $\mathrm{DCO}_{2}=\mathrm{VThf}^{2} \times \mathrm{fR}$. In the last few years, the emergence of volume guarantee $(\mathrm{VG})$ combined with HFOV has allowed independent adjustment of VThf and frequency $[12,13]$. In this way, it is possible to modify the frequency without any effect in the VThf; so, $\mathrm{pCO}_{2}$ decreases as the frequency is increased and the VThf is fixed [14-16]. Furthermore, it has been possible to develop a new theoretical lung protective strategy based on the use of frequencies higher than normally used $(>15 \mathrm{~Hz})$ allowing to reduce the VThf to theoretically minimize volutrauma while maintaining an adequate ventilation (keeping a constant $\mathrm{DCO}_{2}$ and $\mathrm{PCO}_{2}$ ) [17]. To maintain the VThf set at higher frequencies, a higher proximal oscillation pressure amplitude $(\Delta \mathrm{Phf})$ in the circuit could be needed, but it has been demonstrated that distal pressure amplitude does not increase, it may even decrease [18].

So, with this new strategy, very small VThf can be used in a constant way while the frequency is increased to maintain adequate ventilation, and this very small VThf can be protective compared with the standard VThf generated by using lower frequencies.

The aim of the study was to demonstrate the potential protective effect of this new strategy compared with the standard HFOV, by examining histological changes of acute lung injury in a neonatal animal model after 12 hours of ventilation.

\section{Materials and methods}

\section{Study subjects}

Twelve healthy 24- to 48-h old Landrace-Large White piglets were used. Animals were handled according to the European and National Regulations for Protection of Experimental Animals (2010/63/UE and RD 53/2013) and the study was approved by the institutional ethic committee for animal research.

\section{Anesthesia}

General anesthesia was induced via facemask with $8 \%$ sevoflurane in oxygen. Then, anesthesia was maintained with an intravenous constant infusion of propofol as needed. Intraoperative analgesia was achieved with intravenous morphine chlorhydrate $(1 \mathrm{mg} / \mathrm{kg})$, and muscle relaxation was induced with intravenous rocuronium $(1.5 \mathrm{mg} / \mathrm{kg})$ in this animal model to better analyze lung mechanics without the interference of respiratory muscle activity.

\section{Experimental protocol}

When an adequate level of anesthesia was achieved, a tracheostomy was performed and a tracheal tube $(3 \mathrm{~mm}$ inner diameter) was placed and sealed with a ribbon wrapped around the trachea to prevent any leak. Furthermore, by surgical cut down, a 24-gauge polyethylene catheter was placed into the internal jugular vein for continuous infusion of dextrose $5 \%(10 \mathrm{~mL} / \mathrm{kg} / \mathrm{h})$ and the administration of drugs, and a 20-gauge polyethylene catheter was inserted into the right carotid artery, for arterial blood sampling and continuous blood pressure measurement via a calibrated pressure transducer.

Then, the ventilator (Babylog VN500 ${ }^{\circledR}$, Dräger, Lübeck, Germany) was connected to the tracheal tube and adjusted in CMV using Pressure support ventilation (PSV) with VG mode with the following ventilator settings: a positive endexpiratory pressure (PEEP) of $5-6 \mathrm{cmH}_{2} \mathrm{O}$, an inspired oxygen fraction $\left(\mathrm{FiO}_{2}\right)$ of $0.3-0.4$, a frequency of $40-60 \mathrm{rpm}$, and a tidal volume (VT) of 6-7 mL/kg. Fine adjustments were done for a target arterial partial pressure of carbon dioxide $\left(\mathrm{PaCO}_{2}\right)$ of $45-60 \mathrm{~mm} \mathrm{Hg}$ and a target oxygen transcutaneous saturation $\left(\mathrm{SpO}_{2}\right)$ of $90-95 \%$.

Following instrumentation and after a stabilization period of $60 \mathrm{~min}$, bronchoalveolar lavage (BAL) with three aliquots of $10 \mathrm{~mL} / \mathrm{kg}$ isotonic saline solution warmed to body temperature was used to induce surfactant depletion mimicking neonatal respiratory distress syndrome. Lung dynamic compliance (Cdyn) was measured before and after BAL to verify a low compliance situation. Then, $\mathrm{FiO}_{2}$ was increased until 1 and the ventilator was switched to HFOV to carry out a lung volume recruitment maneuver in order to ensure adequate alveolar re-expansion. At first, mean airway pressure (mPaw) was increased from 10 to $25 \mathrm{~cm} \mathrm{H}_{2} \mathrm{O}$ in three 1-min stages. Subsequently, $\mathrm{FiO}_{2}$ was decreased until 0.4 and mPaw was decreased too by $2 \mathrm{~cm} \mathrm{H}_{2} \mathrm{O}$ every $30 \mathrm{~s}$ until $10 \mathrm{~cm} \mathrm{H}_{2} \mathrm{O}$ or $2 \mathrm{~cm} \mathrm{H} \mathrm{H}_{2} \mathrm{O}$ above the critical lung closing pressure (defined as the pressure below which $\mathrm{SpO}_{2}$ decrease below 90\%).

Finally, after lung recruitment was achieved, ventilator settings were adjusted according to the ventilation strategy assigned using random numbers to each group. Three groups were used to better analyze the effect of the two strategies of HFOV and the standard CMV in a similar lung damage situation as a reference.

1. CMV group: Animals assigned to this group were ventilated in CMV using PSV with VG mode with the same ventilator settings described above adjusted for the target $\mathrm{PaCO}_{2}$ of $45-60 \mathrm{~mm} \mathrm{Hg}$. 
2. HFOV groups $(10 \mathrm{~Hz}$ and $20 \mathrm{~Hz}$ HFOV groups): Animals assigned to other two groups were ventilated in $\mathrm{HFOV}$ with VG and adjusted to a mPaw of $10 \mathrm{~cm}$ $\mathrm{H}_{2} \mathrm{O}$, an inspired oxygen fraction of $0.3-0.4$, an IE ratio of $1: 1$, a frequency of 10 or $20 \mathrm{~Hz}$, and the VThf guarantee needed to achieved the target $\mathrm{PaCO}_{2}$ $(45-60 \mathrm{~mm} \mathrm{Hg})$. Delta pressure $(\Delta \mathrm{Phf})$ was adjusted by the ventilator to achieve the set VThf.

A 20-gauge polyethylene catheter was advanced to the carina, between the tracheal tube and the trachea for continuous measurement of mPaw at the trachea (mPaw-t) and $\Delta \mathrm{Phf}$ at the trachea $(\Delta \mathrm{Phf}-\mathrm{t})$. Self-adhering patches were applied to the skin for electrocardiography (ECG) and heart rate recording. Pulse oximetry was recorded continuously by placing a pulse oximeter on the paw, and rectal temperature was also monitored and maintained between $36^{\circ} \mathrm{C}$ and $38^{\circ} \mathrm{C}$ by means of a total temperature management system. Temperature, pulse oximetry, heart rate, arterial blood pressure and airway pressure (referred to a zero level) were registered continuously on a cardiovascular monitor (PM8060 Vitara, Dräger, Lübeck, Germany). Arterial blood was withdrawn anaerobically and immediately analyzed for $\mathrm{pH}$, partial pressure of oxygen $\left(\mathrm{PaO}_{2}\right), \mathrm{PaCO}_{2}$, and arterial oxyhemoglobin saturation $\left(\mathrm{SaO}_{2}\right)$ each $2 \mathrm{~h}$, ensuring an adequate ventilation and oxygenation (IL 1306 $\mathrm{pH} /$ Blood GasAnalyzer, Allied Instrumentation). Data from the ventilator were exported through the standard USB connection.

After $12 \mathrm{~h}$ of ventilation according to the strategy assigned to each group, a new Cdyn measurement was repeated under the same conventional settings on the ventilator. Then, animals were euthanized using a propofol overdose followed by an intravenous bolus of potassium chloride solution $(50 \mathrm{mg} / \mathrm{kg})$.

The HFOV was delivered with a Babylog VN500 ventilator (Dräger, Lübeck, Germany). The Babylog VN500 generates a sinusoidal pressure signal around a set mPaw and has an active inspiration and an active expiration. The VG mode is volume-targeted ventilation where the microprocessor compares the VThf of the previous breath, using leak compensated VThf, and adjusts delta pressure up or down to achieve the set VThf.

\section{Tissue removal and lung processing}

After midline sternotomy, lungs were removed and inflated with saline solution at a pressure of $20 \mathrm{~cm} \mathrm{H}_{2} \mathrm{O}$. Subsequently, lungs were perfused with saline solution and then, the right lung was fixed with formaldehyde $4 \%$. Small pieces from medial lobe of this lung were removed and stored in the fixation solution at $-4{ }^{\circ} \mathrm{C}$. Then, the specimens were embedded in paraffin, sectioned, and stained with hematoxylin and eosin for histological examination.

\section{Histological examination}

Lung histological damage was quantified by a score using five variables (inflammation, edema, hemorrhage, atelectasis, and emphysema). A predefined injury severity was created and graded for each of the variables [19] as shown in Table 1 (total score between 0 and 20). The pathologists were blinded to the assignment group.

\section{Statistical analysis}

Initially, a sample size of six animals per group was considered adequate based on previous studies. An interim analysis was done by the research team once were included four animals per group to prevent extra experiments; as statistically significant differences were observed at this point, only four animals were used per group, in order to reduce the number of animals used according to the European and National Regulations for Protection of Experimental Animals.

Statistical analysis of data was performed using SPSS 15.0 (SPSS Inc., IL, USA). Following confirmation of normal distribution (Shapiro-Wilk test), dynamic compliance was analyzed using a two-way analysis of variance (ANOVA) for repeated measures followed by Tukey's test for multiple comparisons. The mean VThf in HFOV groups was analyzed using a $t$-test for unpaired data. These data are presented as means \pm standard deviation. For

Table 1 Defined lung histological examination score

Inflammation

Edema, hemorrhage, atelectasis, emphysema

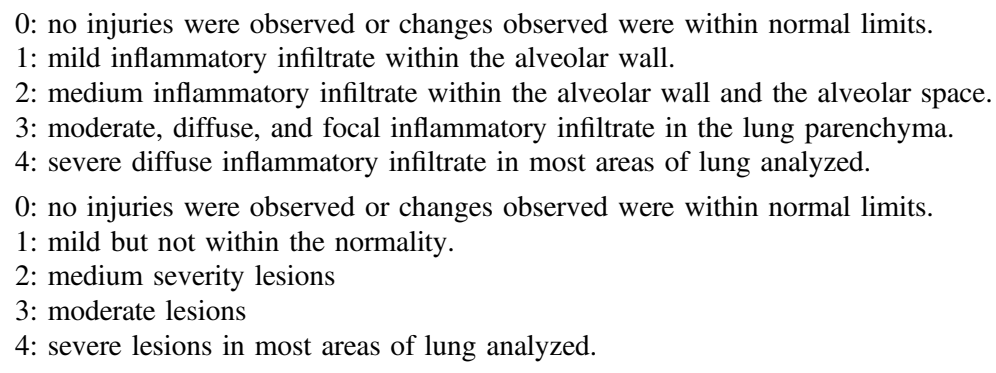

1: mild inflammatory infiltrate within the alveolar wall

3: moderate, diffuse, and focal inflammatory infiltrate in the lung parenchyma.

4: severe diffuse inflammatory infiltrate in most areas of lung analyzed.

1: mild but not within the normality.

2: medium severity lesions

4: severe lesions in most areas of lung analyzed. 


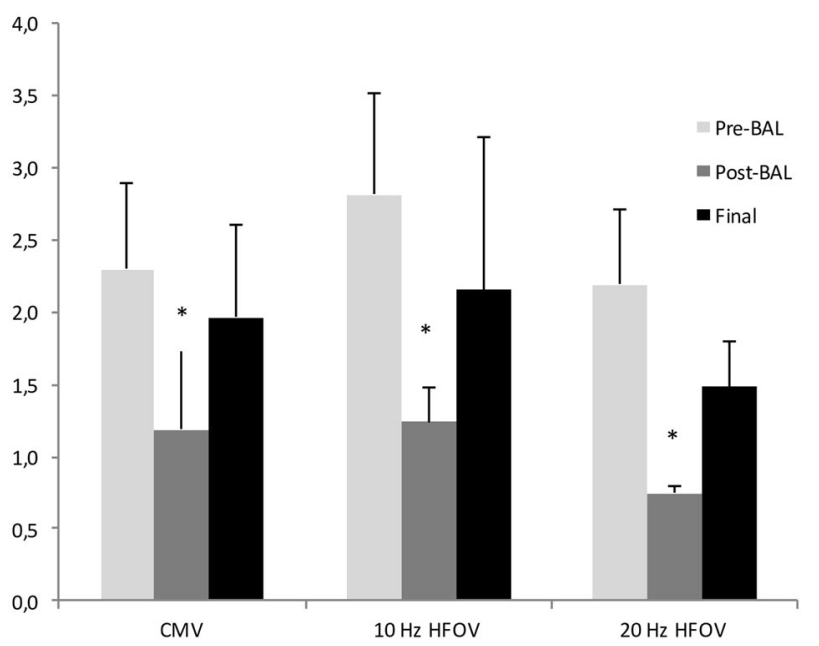

Fig. 1 Dynamic respiratory compliance (Cdyn) pre-BAL, post-BAL, and final. Cdyn was significantly lower in post-BAL compared with pre-BAL for CMV $\left(2.29 \mathrm{ml} / \mathrm{cm} \mathrm{H}_{2} \mathrm{O}\right.$ vs $\left.1.19 \mathrm{ml} / \mathrm{cm} \mathrm{H}_{2} \mathrm{O} ; p=0.001 *\right)$, $10 \mathrm{~Hz}\left(2.81 \mathrm{ml} / \mathrm{cm} \mathrm{H}_{2} \mathrm{O}\right.$ vs $\left.1.24 \mathrm{ml} / \mathrm{cm} \mathrm{H}_{2} \mathrm{O} ; p=0.035^{*}\right)$, and $20 \mathrm{~Hz}$ groups $\left(2.19 \mathrm{ml} / \mathrm{cm} \mathrm{H}_{2} \mathrm{O}\right.$ vs $\left.0.75 \mathrm{ml} / \mathrm{cm} \mathrm{H}_{2} \mathrm{O} ; p=0.006 *\right)$. BAL bronchoalveolar lavage, $\mathrm{CMV}$ conventional mechanical ventilation, HFOV high-frequency oscillatory ventilation. ${ }^{*} p<0.05$

non-parametric data (lung histological score comparison), an ANOVA of Kruskall-Wallis was used. Statistical significance was accepted when $p<0.05$.

\section{Results}

The mean ( \pm standard deviation, SD) body weight of the piglets were $2.57 \pm 0.59 \mathrm{~kg}$. All animals were adequately ventilated and oxygenated. There were no significant differences in hemodynamic parameters between groups.

Cdyn was measured pre- and post-BAL, as well as at the end of the study. As shown in Fig. 1, BAL significantly reduced Cdyn in a similar way in all the groups. At the end of the experiment, Cdyn improved in all groups.

\section{CMV group}

Piglets in the CMV group needed a VT of $7.33 \pm 0.20 \mathrm{ml} / \mathrm{kg}$ to achieve the target $\mathrm{PaCO}_{2}$ of $45-60 \mathrm{~mm} \mathrm{Hg}$.

Emphysema was the main lesion in this group. Inflammatory infiltrate and atelectasis were also found in this group. Neither hemorrhage nor edema were found in the animals of this group (Table 2 and Fig. 2).

\section{HFOV groups}

Piglets in the $20 \mathrm{~Hz}$ group needed a lower VThf than those in the $10 \mathrm{~Hz}$ group to achieve the target $\mathrm{PaCO}_{2}$ of $45-60 \mathrm{~mm} \mathrm{Hg}(1.56 \mathrm{ml} / \mathrm{kg}$ vs $2.54 \mathrm{ml} / \mathrm{kg} ; p=0.004)$.
Table 2 Lung histological examination of HFOV and CMV groups

\begin{tabular}{|c|c|c|c|c|c|c|c|c|c|c|c|c|}
\hline \multirow[b]{2}{*}{ Animal case } & \multicolumn{4}{|c|}{$10 \mathrm{~Hz}$ group } & \multicolumn{4}{|c|}{$20 \mathrm{~Hz}$ group } & \multicolumn{4}{|c|}{ CMV group } \\
\hline & I & II & III & IV & $\mathrm{V}$ & VI & VII & VIII & IX & $\mathrm{X}$ & $\mathrm{XI}$ & XII \\
\hline Inflammation & 3 & 2 & 4 & 3 & 1 & 2 & 0 & 0 & 1 & 1 & 1 & 3 \\
\hline Hemorrhage & 1 & 1 & 3 & 0 & 0 & 0 & 0 & 0 & 0 & 0 & 0 & 0 \\
\hline Atelectasis & 2 & 2 & 4 & 3 & 2 & 3 & 1 & 2 & 1 & 1 & 1 & 3 \\
\hline Edema & 0 & 0 & 0 & 0 & 0 & 0 & 0 & 0 & 0 & 0 & 0 & 0 \\
\hline Emphysema & 0 & 0 & 0 & 0 & 0 & 0 & 0 & 0 & 3 & 3 & 0 & 2 \\
\hline Total score & 28 & & & & 11 & & & & 20 & & & \\
\hline
\end{tabular}

HFOV high-frequency oscillatory ventilation, $C M V$ conventional mechanical ventilation

More evident and more severe histological lesions were found in the $10 \mathrm{~Hz}$ group with the higher VThf. Inflammatory infiltrate was the main lesion in this group, including mononuclear infiltrate of lymphocytes, plasma cells, and macrophages within the alveolar wall and alveolar space, as well as large areas of parenchyma consolidation. Areas of alveolar hemorrhage were found in the more severe cases. Finally, areas of atelectasis were found in most cases. The more severe the inflammation and hemorrhage, the more severe atelectasis was found. Edema and emphysema were not found in this group.

The number of samples with inflammatory infiltrate, as well as its severity in the $20 \mathrm{~Hz}$ groups was less than in the $10 \mathrm{~Hz}$ group. Atelectasis was the main lesion in the $20 \mathrm{~Hz}$ group with the lower VThf, but mild or medium. No hemorrhage, edema, and emphysema were found in this group (Table 2 and Fig. 2).

When lung histological score was compared between groups, statistically significant differences were observed for inflammation $(p=0.043)$, hemorrhage $(p=0.027)$, and emphysema $(p=0.027)$.

\section{Discussion}

We have demonstrated a reduced lung injury histological score with the use of a strategy of lower compared with higher VThf on HFOV combined with VG in an animal neonatal model of lung injury during mechanical ventilation. It has become increasingly evident that lung overdistension by the use of large gas volume is an important factor to induce VILI [20]. Furthermore, Wakabayashi et al. demonstrated that lung-marginated, intravascular monocytes are only activated following lung overstretch, not atelectasis, leading to proinflammatory cytokines production that promote VILI and nonpulmonary organs injury ("biotrauma") [21]. So, the use of strategies to reduce as much as possible the tidal volume may minimize lung and systemic injury. 
Fig. 2 Lung histological examination. a, b $10 \mathrm{~Hz}$ highfrequency oscillatory ventilation (HFOV) group. Severe diffuse inflammatory infiltrate and hemorrhage. c, d $20 \mathrm{~Hz} \mathrm{HFOV}$ group. Medium atelectasis. e, f Conventional mechanical ventilation (CMV) group. Moderate emphysema with large alveolar rupture
A

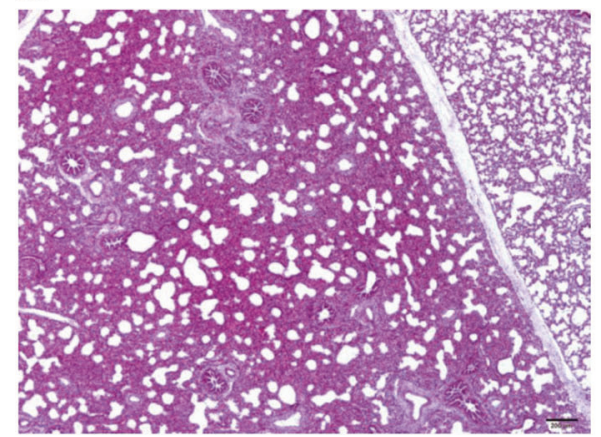

C

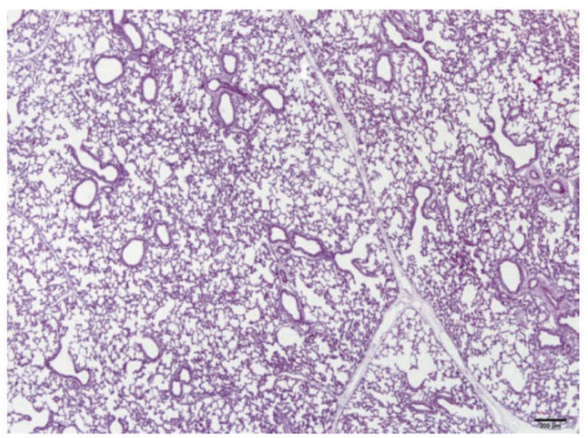

$\mathbf{E}$

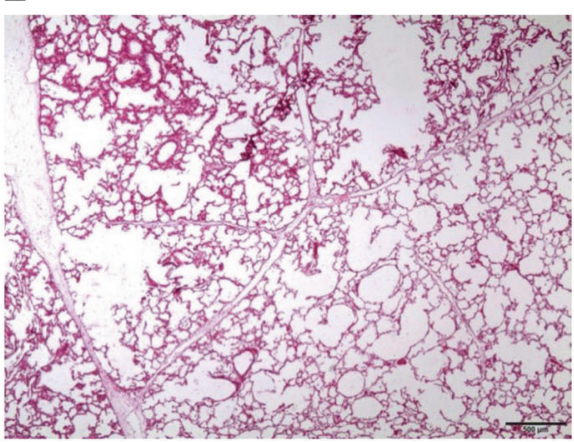

B

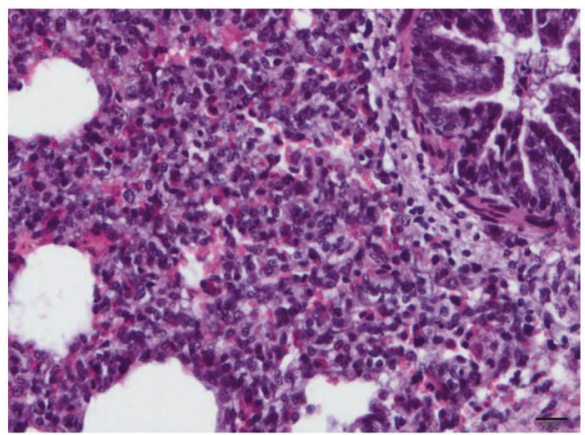

D

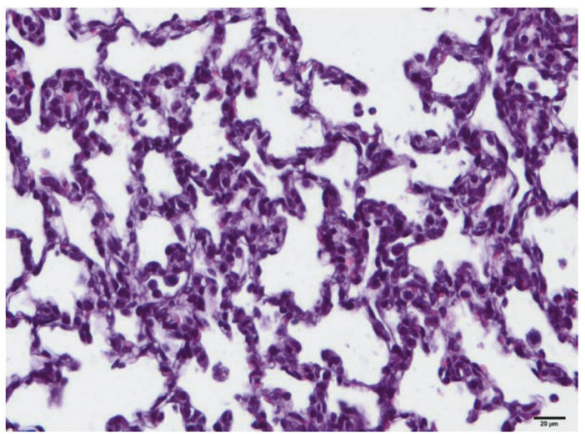

F

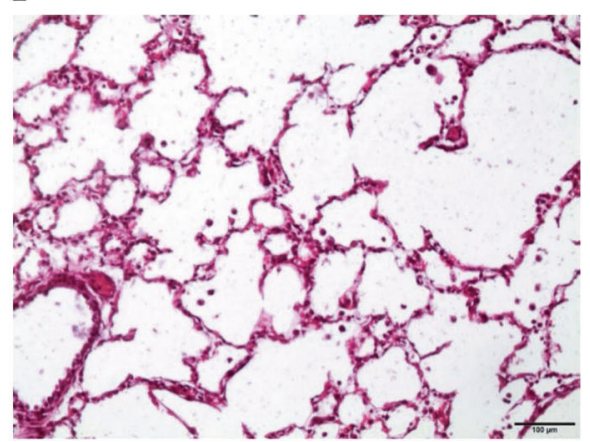

In contrast to HFOV, emphysema was the main lesion in CMV group; inflammatory infiltrate and atelectasis were also found in this group, but milder. Previous experimental models that compared both, HFOV without VG at $10 \mathrm{~Hz}$ [22] and $15 \mathrm{~Hz}$ [23] and CMV using a protection ventilator strategy with low tidal volume $(5-6 \mathrm{~mL} / \mathrm{kg})$ and high PEEP $\left(8-10 \mathrm{~cm} \mathrm{H}_{2} \mathrm{O}\right.$ ) have shown that the use of HFOV was associated with an attenuation of lung inflammation (polymorphonuclear infiltration and tumor necrosis factor- $\alpha$ production in the alveolar space), histopathological changes of the small airways and alveoli and oxidative lung injury. These authors suggested that a more homogeneous lung inflation due to an adequate lung volume recruitment in HFOV may limit alveolar stretch minimizing VILI. No significant differences of inflammation were found between $20 \mathrm{~Hz}$ HFOV and CMV groups in our model; however, no emphysema was found in $20 \mathrm{~Hz} \mathrm{HFOV}$ group compared with the CMV group and the degree of lung histological damage was greater in CMV group based on our developed score (20 points vs 11 points). We hypothesize that the lower inflation pressure at the alveoli, the minor variations in pressure and volume and the relatively constant lung volume above the functional residual capacity achieved in HFOV compared with CMV, may explain these differences.

We found that when a higher VThf was used in HFOV, there was more evident and severe inflammatory infiltrate, including mononuclear infiltrate of lymphocytes, plasma cells, and macrophages within the alveolar wall and alveolar space, as well as large areas of parenchyma consolidation. Meyer et al. compared lung lesions resulting from two different HFOV regimes in a rabbit model, at $5 \mathrm{~Hz}$ and $15 \mathrm{~Hz}$ without the combined use of VG, so the higher the frequency, the lower the VThf. They observed a significantly lower neutrophil count within alveoli in animals ventilated with the higher frequency regime (and lower VThf) after $4 \mathrm{~h}$ [24]. Furthermore, in a large animal model 
of acute respiratory distress syndrome, HFOV at higher frequencies $(9 \mathrm{~Hz}$ vs 3 and $6 \mathrm{~Hz})$ delivered smaller tidal volumes and minimized stress on the lung by reducing lung injury and the expression of inflammatory mediators. Lung histopathology revealed less hemorrhage and neutrophil infiltration in the alveoli and interstitium and less interstitial lymphocyte infiltration in animals ventilated under HFOV at $9 \mathrm{~Hz}$ and smaller tidal volumes, compared with lower frequencies, as we showed in our model [25].

So, based on our results, the use of very low tidal volume in HFOV combined with VG, together with an adequate lung volume recruitment, may reduce lung damage in immature infants.

The main limitation of our model was the short duration of the ventilator strategy used, $12 \mathrm{~h}$, which only shows the acute lung injury. However, it is well known that inflammation occurs shortly after birth, driven by antenatal factors in combination with early postnatal exposure to intensive care interventions such us mechanical ventilation; and that this early inflammation plays a critical role in the development of BPD [26]. Thus, any intervention used to reduce lung inflammation in this vulnerable period may be effective to reduce lung damage and BPD. Furthermore, the neonatal animal model used in this study is not defined to reproduce BPD, but it is useful to analyze acute lung damage of mechanical ventilation.

In conclusion, the use of very low tidal volume with increasing frequencies in HFOV combined with VG reduces inflammatory response and lung histological damage. So, this new protective lung strategy may allow minimizing VILI in immature infants in the immediate neonatal period.

Acknowledgements The authors thank Dräger Medical GmbH for providing the Babylog VN500 for the present study.

Funding This study was supported in part by the National Grant of the Instituto de Salud Carlos III and the Fondo Europeo de Desarrollo Regional, FIS 14/00149.

\section{Compliance with ethical standards}

Conflict of interest MS-L has received advisory board consulting fees from Dräger. The remaining authors declare that they have no conflict of interest.

Publisher's note: Springer Nature remains neutral with regard to jurisdictional claims in published maps and institutional affiliations.

\section{References}

1. Coalson JJ. Pathology of bronchopulmonary dysplasia. Semin Perinatol. 2006;30:179-84.

2. Gupta S, Sinha SK, Donn SM. Ventilatory management and bronchopulmonary dysplasia in preterm infants. Semin Fetal Neonatal Med. 2009;14:367-73.
3. Jobe $\mathrm{AH}$, Ikegami M. Lung development and function in preterm infants in the surfactant treatment era. Annu Rev Physiol. 2000;62:825-46.

4. Slutsky AS. Lung injury caused by mechanical ventilation. Chest . 1999;116(1 Suppl):9S-15S.

5. Ambalavanan N, Carlo WA. Ventilatory strategies in the prevention and management of bronchopulmonary dysplasia. Semin Perinatol. 2006;30:192-9.

6. The Acute Respiratory Distress Syndrome Network. Ventilation with lower tidal volumes as compared with traditional tidal volumes for acute lung injury and the acute respiratory distress syndrome. N Eng J Med. 2000;342:1301-8.

7. Froese AB, Kinsella JP. High-frequency oscillatory ventilation: lessons from the neonatal/pediatric experience. Crit Care Med. 2005;33:S115-21.

8. Hamilton PP, Onayemi A, Smyth JA, Gillan JE, Cutz E, Froese $\mathrm{AB}$, et al. Comparison of conventional and high-frequency ventilation: oxygenation and lung pathology. J Appl Physiol Respir Environ Exerc Physiol. 1983;55:131-8.

9. Meredith KS, deLemos RA, Coalson JJ, King RJ, Gerstmann DR, Kumar R, et al. Role of lung injury in the pathogenesis of hyaline membrane disease in premature baboons. J Appl Physiol (1985). 1989;66:2150-8.

10. Cools F, Offringa M, Askie LM. Elective high frequency oscillatory ventilation versus conventional ventilation for acute pulmonary dysfunction in preterm infants. Cochrane Database Syst Rev. 2015;3: CD000104. https://doi.org/10.1002/14651858.CD000104.pub4

11. Leipälä JA, Iwasaki S, Milner A, Greenough A. Accuracy of the volume and pressure displays of high frequency oscillators. Arch Dis Child Fetal Neonatal Ed. 2004;89:F174-6.

12. Sánchez Luna M, Santos González M, Tendillo Cortijo F. Highfrequency oscillatory ventilation combined with volume guarantee in a neonatal animal model of respiratory distress syndrome. Crit Care Res Pract. 2013;2013:593915. https://doi.org/10.1155/2013/593915

13. Iscan B, Duman N, Tuzun F, Kumral A, Ozkan H. Impact of volume guarantee on high-frequency oscillatory ventilation in preterm infants: a randomized crossover clinical trial. Neonatology . 2015;108:277-82.

14. Kamitsuka MD, Boynton BR, Villanueva D, Vreeland PN, Frantz ID. Frequency, tidal volume, and mean airway pressure combinations that provide adequate gas exchange and low alveolar pressure during high frequency oscillatory ventilation in rabbits. Pediatr Res. 1990;27:64-9.

15. Slutsky AS, Kamm RD, Rossing TH, Loring SH, Lehr J, Shapiro $\mathrm{AH}$, et al. Effects of frequency, tidal volume, and lung volume on $\mathrm{CO}_{2}$ elimination in dogs by high frequency $(2-30 \mathrm{~Hz})$, low tidal volume ventilation. J Clin Invest. 1981;68:1475-84.

16. Watson JW, Jackson AC, Gillespie JR. $\mathrm{CO}_{2}$ elimination and airway opening pressure during high frequency oscillation in dogs. Respir Physiol. 1984;58:235-44.

17. González-Pacheco N, Sánchez-Luna M, Ramos-Navarro C, Navarro-Patiño $\mathrm{N}$, de la Blanca AR-S. Using very high frequencies with very low lung volumes during high-frequency oscillatory ventilation to protect the immature lung. A pilot study. J Perinatol. 2016;36:306-10.

18. Mukerji A, Belik J, Sanchez-Luna M. Bringing back the old: time to reevaluate the high-frequency ventilation strategy. J Perinatol. 2014;34:464-7.

19. Mann PC, Vahle J, Keenan CM, Baker JF, Bradley AE, Goodman DG, et al. International Harmonization of toxicologic pathology nomenclature: an overview and review of basic principles. Toxicol Pathol. 2012;40:7S-13S.

20. Slutsky AS. Ventilator-induced lung injury: from barotrauma to biotrauma. Respir Care. 2005;50:646-59.

21. Wakabayashi K, Wilson MR, Tatham KC, O'Dea KP, Takata M. Volutrauma, but not atelectrauma, induces systemic cytokine 
production by lung-marginated monocytes. Crit Care Med. 2014;42:e49-57.

22. Ronchi CF, dos Anjos Ferreira AL, Campos FJ, Kurokawa CS, Carpi MF, de Moraes MA, et al. High-frequency oscillatory ventilation attenuates oxidative lung injury in a rabbit model of acute lung injury. Exp Biol Med. 2011;236:1188-96.

23. Imai Y, Nakagawa S, Ito Y, Kawano T, Slutsky AS, Miyasaka K. Comparison of lung protection strategies using conventional and high-frequency oscillatory ventilation. J Appl Physiol. 2001;91:1836-44.
24. Meyer J, Cox PN, McKerlie C, Bienzle D. Protective strategies of high-frequency oscillatory ventilation in a rabbit model. Pediatr Res. 2006;60:401-6.

25. Liu S, Yi Y, Wang M, Chen Q, Huang Y, Liu L, Xie J, et al. Higher frequency ventilation attenuates lung injury during high-frequency oscillatory ventilation in sheep models of acute respiratory distress syndrome. Anesthesiology. 2013;119:398-411.

26. Leroy S, Caumette E, Waddington C, Hébert A, Brant R, Lavoie PM. A time-based analysis of inflammation in infants at risk of bronchopulmonary dysplasia. J Pediatr. 2018;192:60-5. 